\title{
Rapid quantification of stroke volume using magnetohydrodynamic voltages in 3T MRI: a feasibility study
}

\author{
Stan T Gregory ${ }^{1 *}$, Ehud J Schmidt ${ }^{2}$, Shelley H Zhang ${ }^{2}$, Raymond Y Kwong ${ }^{2}$, William G Stevenson², John Oshinski ${ }^{3}$, \\ Zion $\mathrm{T} \mathrm{Tse}^{1}$
}

From 18th Annual SCMR Scientific Sessions

Nice, France. 4-7 February 2015

\section{Background}

The Electrocardiogram (ECG) is a standard clinical tool for cardiac physiological monitoring, required for cardiac synchronization during cardiac MRI, despite signal artifacts resulting from Magnetohydrodynamic voltages (VMHD). VMHD becomes significant during systole when rapidly ejected blood from the left ventricle into the aortic arch interacts with the strong magnetic field $\left(\mathrm{B}_{0}\right)$ of the MRI [1]. Due to this relationship, we hypothesized that blood flow as a function of time in the cardiac cycle and left ventricular Stroke Volume (SV) could be derived using VMHD extracted from intra-MRI ECG. This method would allow for real-time beat-to-beat SV estimation during clinical MR scanning and cardiac MRI stress testing. This non-invasive real-time physiological measure of patient condition can be provided with the described software processing during conventional cardiac MRI routines, and potentially replace Invasive Blood Pressure during complex interventional procedures.

\section{Methods}

Velocity-Encoded (VENC) Phase Contrast Cine MRI slices were obtained in three healthy volunteer subjects $(n=3)$ along the aortic arch (Fig. 1a) to quantify the volume of blood flow and SV using a Siemens Skyra 3T MRI scanner with the following scan parameters: VENC: $150 \mathrm{~cm} / \mathrm{s}$; TR: $44.08 \mathrm{~ms}$; TE: $3.28 \mathrm{~ms}$; and Flip Angle: $20^{\circ}$. A GE digital-IT 12-Lead ECG recording system modified to be MRI-compatible [2,3] was used to record 12-lead ECG traces from the subjects at $3 \mathrm{~T}$ (Fig. 1b-c). VMHD voltages at each electrode were extracted through the subtraction of ECGs obtained with the subjects outside and inside the MRI [2]. The 12-lead VMHD traces were converted into the Vectorcardiograms (VCG) frame of reference using an inverse Dower transform [4]. For each subject, a multiple linear regression (MLR) (Fig. 1d) was used to correlate VMHD in the VCG reference frame (Fig. 1e) to blood flow volume as a function of time obtained using Cine PC MRI scans over 33 cardiac cycles (Fig. 1f-g). VMHD-derived blood flow velocity was then time-integrated over the systolic cardiac phase to estimate SV, and compared to the "goldstandard" derived from PC MRI.

\section{Results}

After MLR was performed for each subject (Table 1), aortic blood flow as a function of time was computed from the VMHD(t) and compared through VENC PC MRI to evaluate fit, with correlation determined through a Spearman's Ranked Coefficient, found to be $>0.84$ (Fig. 1f). VMHD-based SV was determined with a $<3.6 \%$ error as compared to PC MRI in all three subjects. Fig. 1g shows the real-time beat-to-beat blood flow velocity and SV derived from VMHD, as well as the associated Heart Rate (HR) and raw ECG channel (Precordial Lead V6).

\section{Conclusions}

Relatively accurate beat-to-beat stroke volume and blood flow velocity estimates can be obtained from MHD voltages extracted from 12-lead ECG, providing a means for enhanced patient monitoring inside the MRI bore. A relatively short Phase-Contrast Cine measurement is required to provide the required patient-specific parameters.

University of Georgia, Athens, GA, USA

Full list of author information is available at the end of the article 


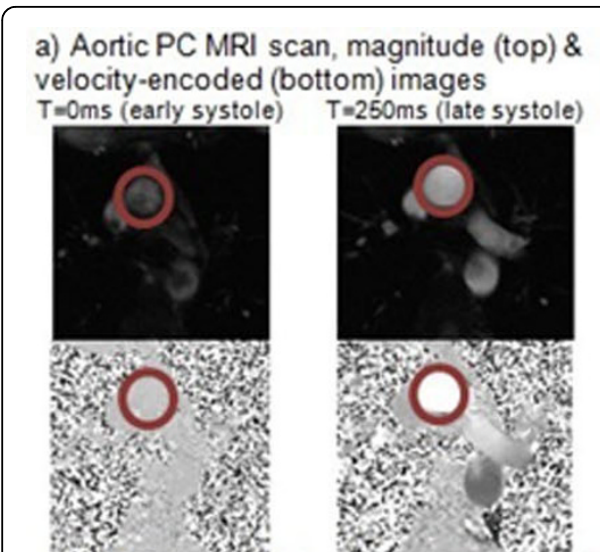

d) VMHD-derived blood flow velocity equation as a function of the directional MHD voltages in the VCG frame of reference,

(Eq. 1), where $A_{0}, A_{1}, A_{2}, \& A_{3}$ are experimentally derived using MLR and Analysis of Variance (ANOVA) fitting methods. SV is derived from the systolic-time-integrated-blood flow velocity (Eq. 2)

$$
\text { Eq. 1: FlowVelocity }(t)_{V M H D}=A_{0}+A_{1} \times V M H D(t)_{X}
$$$$
+A_{2} \times V M H D(t)_{Y}+A_{3} \times V M H D(t)_{Z}
$$

Eq. 2: SV $=\int_{S y s t o l i c \text { Phase }}^{\square \text { FlowVelocity }} y_{V M D} d t \cong$

$$
\begin{aligned}
\int_{\text {Systolic Phase }}^{\square}\left(A_{0}\right. & +A_{1} \times V M H D_{X}+A_{2} \times V M H D_{Y} \\
& +A_{3} \times V M H D_{z} d t
\end{aligned}
$$

f) MRI \& VMHD derived blood flow velocities (blue) alongside extracted VMHD in the VCG reference frame (green)

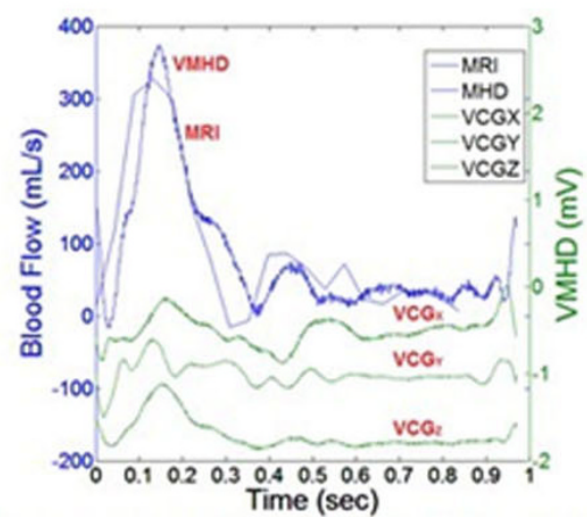

c) 12-lead ECG acquired inside the MRI

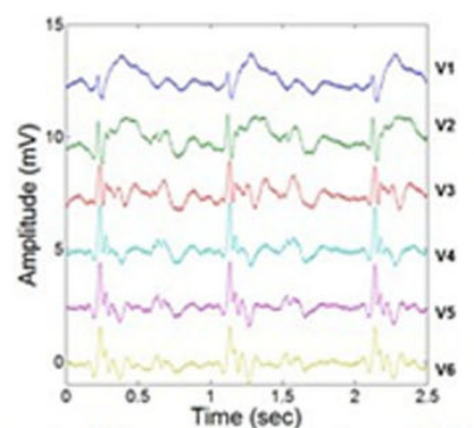

e) Body planes shown in MRI coordinate system: X-Y is shown to be the transverse plane, $X-Z$ is the coronal plane, and $Y-Z$ is the sagittal plane for human subjects in the MRI bore. 


\section{Funding}

NIH U41-RR019703, NIH R03 EB013873-01A1, SBIR-1

R43 HL110427-01.

\section{Authors' details}

'University of Georgia, Athens, GA, USA. 'Brigham and Women's Hospital,

Boston, MA, USA. ${ }^{3}$ Emory University Hospital, Atlanta, GA, USA.

Published: 3 February 2015

\section{References}

1. Gupta: IEEETransBioMedEng 2008.

2. Tse: MRM 2013.

3. Gregory: MRM 2014

4. Dower: CC 1980

doi:10.1186/1532-429X-17-S1-P32

Cite this article as: Gregory et al: Rapid quantification of stroke volume using magnetohydrodynamic voltages in 3T MRI: a feasibility study. Journal of Cardiovascular Magnetic Resonance 2015 17(Suppl 1):P32.

Submit your next manuscript to BioMed Central and take full advantage of:

- Convenient online submission

- Thorough peer review

- No space constraints or color figure charges

- Immediate publication on acceptance

- Inclusion in PubMed, CAS, Scopus and Google Scholar

- Research which is freely available for redistribution

Submit your manuscript at www.biomedcentral.com/submit
() Biomed Central 